\title{
Editorial
}

\section{Geophysical Methods for Environmental Studies}

\author{
Umberta Tinivella, ${ }^{1}$ Michela Giustiniani, ${ }^{1}$ and Giorgio Cassiani ${ }^{2}$ \\ ${ }^{1}$ Istituto Nazionale di Oceanografia e di Geofisica Sperimentale, Borgo Grotta Gigante 42C, 34010 Trieste, Italy \\ ${ }^{2}$ University of Padua, via Gradenigo 6, 35127 Padua, Italy \\ Correspondence should be addressed to Umberta Tinivella; utinivella@ogs.trieste.it
}

Received 2 May 2013; Accepted 2 May 2013

Copyright (c) 2013 Umberta Tinivella et al. This is an open access article distributed under the Creative Commons Attribution License, which permits unrestricted use, distribution, and reproduction in any medium, provided the original work is properly cited.

Geophysical methods are powerful tools to explore the subsoil for environmental investigations. Among the possible fields of application are hydrological and hydrogeological characterization and monitoring, slope stability assessments, archaeological surveys, locating voids and karstic features, soil characterization, contamination assessment, and so forth. Ground-penetrating radar (GPR), seismics, DC resistivity, electromagnetic induction, gravity, and magnetic methods are the most used, whilst the use of other technologies has grown over the past decades. The available techniques are characterised by different penetration and resolution capabilities, from few centimeters to kilometers. For this reason, there is drive in the scientific community towards the integration of several geophysical methods in order to detect and characterize the subsoil from the analysis of different physical properties.

A paper in this special issue presents an original use of the gravity field to model the sea level oscillations. The analysis of the gravity field leads to the forecast of sea level variation due to meteorological tide events. This analysis defines a compensation in the inverse hydrobarometric factor to be taken into account for a correct compensation of the atmospheric pressure variations in basins. This phenomenon has several consequence in Harbor Water Side Management and in Water Quality Control as shown by the reported case studies and introduces a new reference parameter (the socalled Water 1000).

The main topic of the paper authored by Y. Nicaise et al. is the use of electrical resistivity and electromagnetic conductivity methods to demonstrate that geophysical methods can greatly contribute to the study of the water table mineralization in the littoral zone for a sustainable management of water resources. In fact, this study allows the determination of the spatial extent of the water table mineralization by direct measurements of water conductivity in wells and the depth of the fresh/saline water interface in the coastal aquifer by means of geophysical measurements. Moreover, the electromagnetic measurements allow to map a gradient of water salinity in the North West direction. On the environmental level, they have demonstrated that these methods can be used in the zones where drilling of the wells is difficult even impossible or useless in order to delimit the zones where the water resource is exploitable.

The paper of A. Al-Zoubi et al. is focused on microgravity investigations that are a powerful tool for subsurface imaging and especially for the localization of underground karsts. Several geological, technical, and environmental factors, such as the influence of regional trends, interfere with microgravity survey processing and interpretation. A. Al-Zoubi et al. observe that the most effective methods for regional trend removal are the bilinear saddle and local polynomial regressions. Application of these methods makes it possible to detect the anomalous gravity effects from buried targets and to extract the local gravity anomaly at the study site. Consequently, the 3D gravity field modeling led to the identification of the parameters of a physical-geological model in the Eastern Dead Sea coast (Jordan).

The study area of S. Keydar et al. is again the Dead Sea area. In particular, the authors investigate the fast decline of the Dead Sea level, as a possible result of extensive human activities. The authors analyse 3D high resolution seismic data. The method is based on a spatial summation of seismic waves along time surfaces using recently proposed multipath summation with proper weights. This approach 
does not require any explicit information on parameters, since the involved multipath summation is performed for all possible parameter values within a wide specified range. The results from processed 3D time volume show subhorizontal coherent reflectors at an approximate depth of 50-80 m which show dip changes in proximity to outcropping sinkholes and suggest a possible link between revealed fault and the sinkholes.

The paper by Simonenko proposes a possible cosmic energy gravitational genesis of the strong Chinese 2008 and the strong Japanese 2011 earthquakes, based on the established generalized differential formulation of the first law of thermodynamics.

Finally, Romanova et al. examine all worldwide launch crashes occurring at all major 31 launching sites from 1957 to 2008. The authors have developed a comprehensive database, which includes 50 years of information from the beginning of the space age in 1957. The authors analyze correlations with the geomagnetic indices as indirect indicators of the space weather condition. In view of the high costs associated with delaying launch and possibly missing launch windows, only a nowcast could provide a sufficiently reliable service. Thus, space weather real-time monitoring and prediction could become an important issue in the optimization of launch procedures.

In conclusion, the papers reported in this special issue contribute to highlight the role of integrated geophysical methods, illustrating technologies and methods of data analysis that can be applied and developed in various areas of public interest. Moreover, the papers set the stage for emerging future topics in this important interdisciplinary field.

We would like to dedicate this special issue to Ilaria Sanvenero, a brilliant physics student at the University of Pisa, who passed away far too soon at the age of twenty-two. Here, we wish to remember Ilaria by paying a public homage to her brief but precious work. Her thesis has been part of a paper in this special issue presented by Faggioni et al., who will preserve the memory of this wonderful girl in their heart.

Umberta Tinivella

Michela Giustiniani

Giorgio Cassiani 

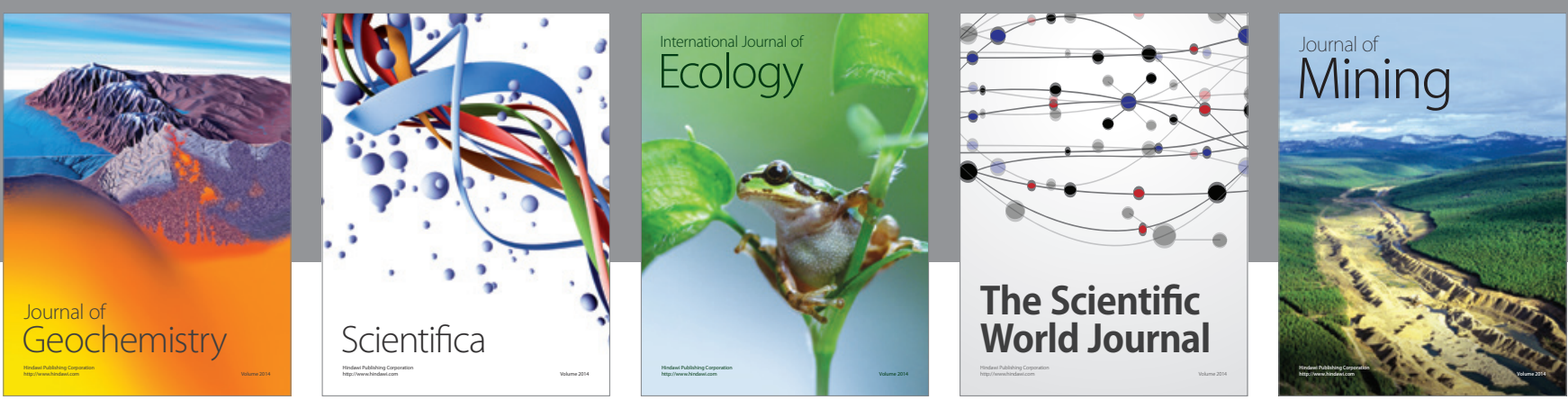

The Scientific World Journal
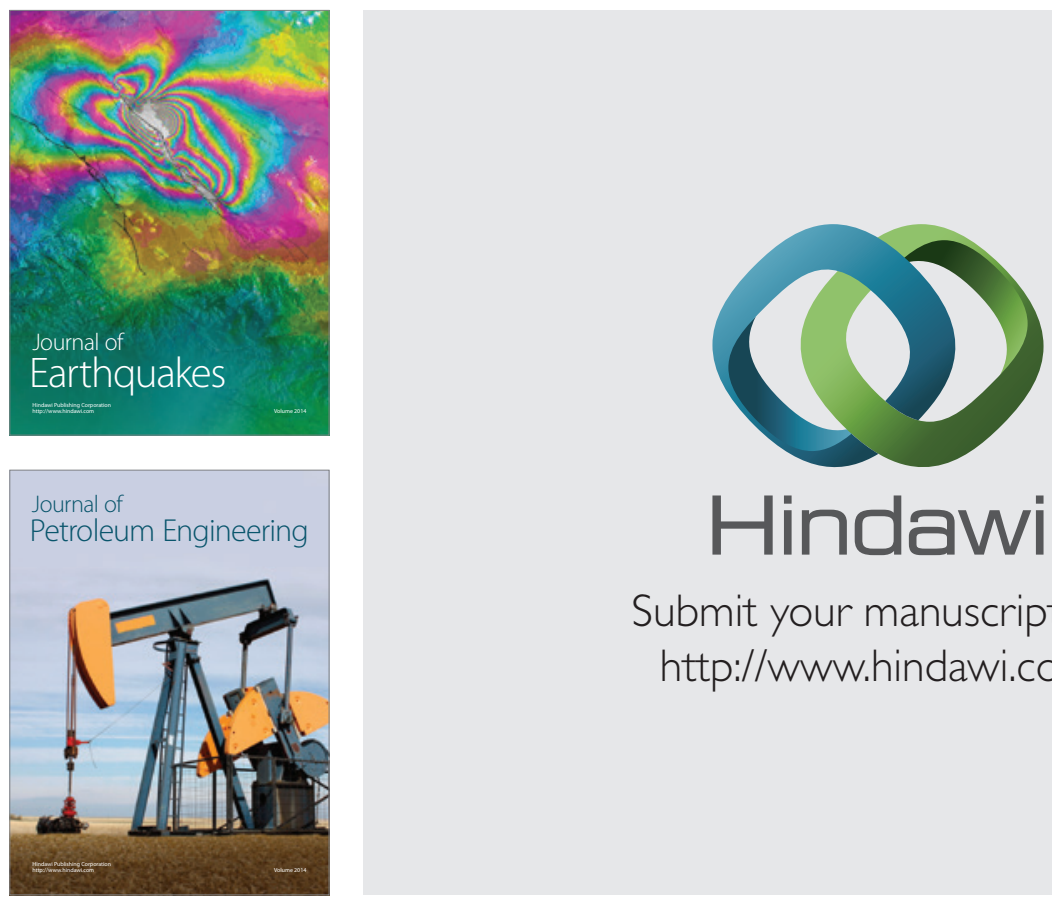

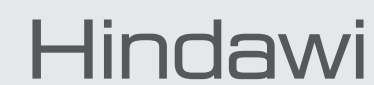

Submit your manuscripts at

http://www.hindawi.com
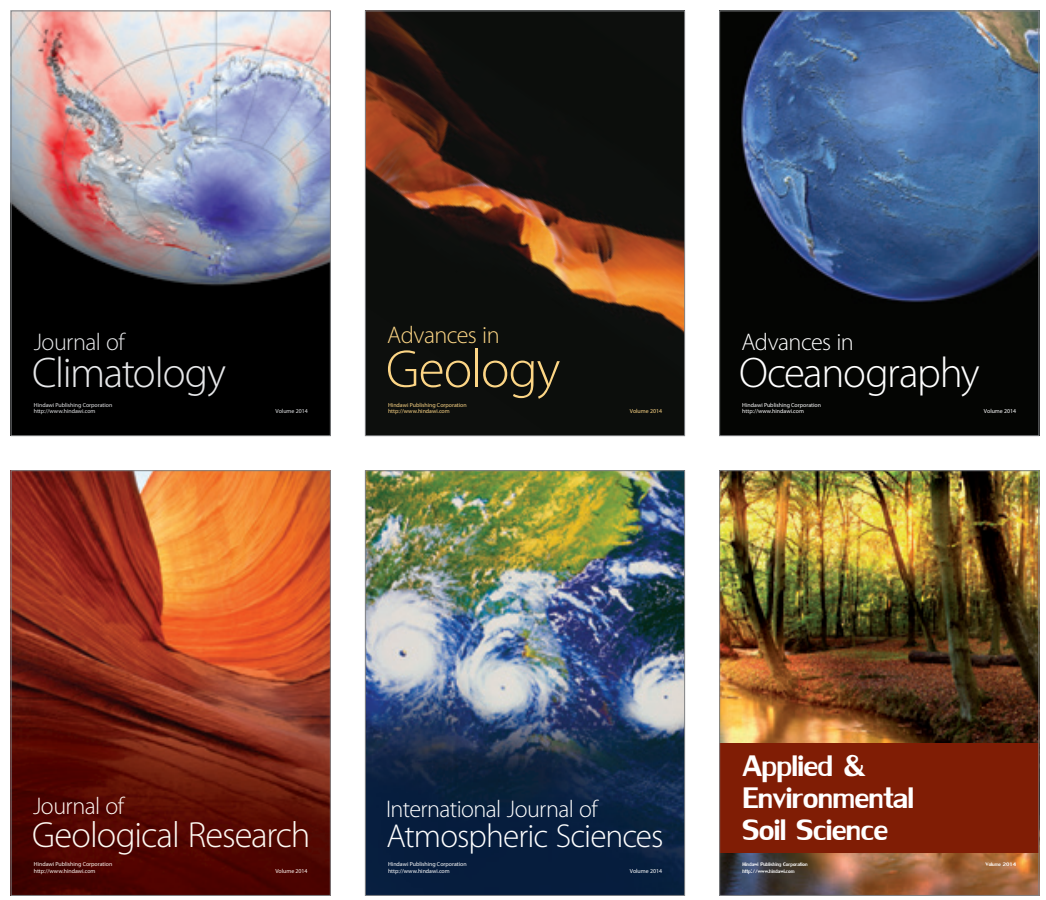
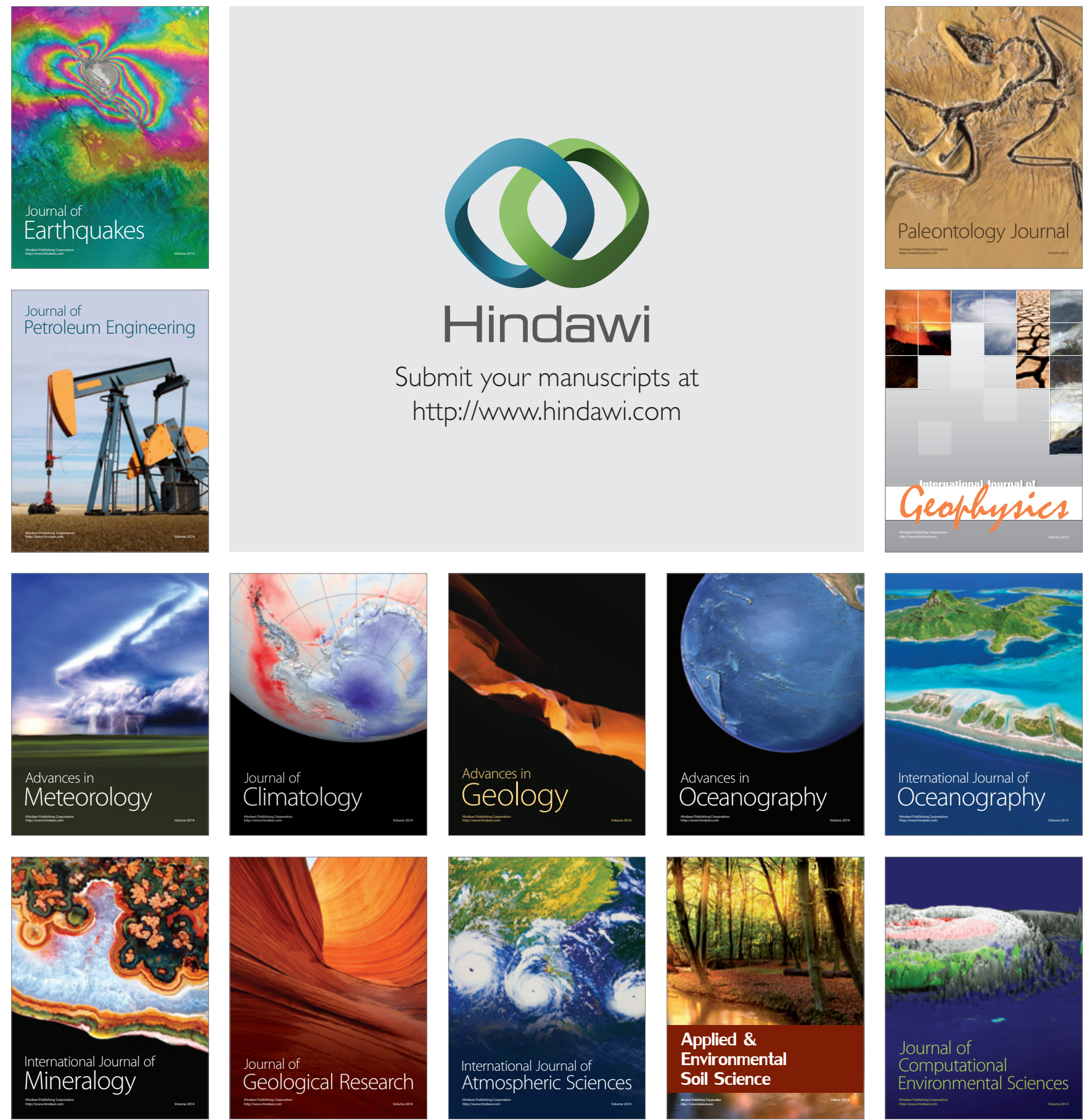